\title{
Maternity Nurses' Perception Regarding Challenges Facing Ten Steps to Successful Breastfeeding Implementation: Qualitative Research Approach
}

\author{
Eman Ahmed Elkaluby ${ }^{1} \quad$ Neama Yousef Mohamed ${ }^{2 *}$ \\ 1. Lecturer, Community Health Nursing, Faculty of Nursing, Alexandria University, Egypt \\ 2.Assistant professor, Community Health Nursing, Faculty of Nursing, Alexandria University, Egypt
}

\begin{abstract}
Background: Breastfeeding is one of the most effective ways to ensure child health and survival. It is also a critical key to achieve many of the Sustainable Development Goals (SDGs). Promotion, supporting and protecting breastfeeding has been a mandate of the global strategy of Infant and Young Child Feeding (IYCF). The implementation of such strategy is intensified by positive nurses' perception and commitment to do so. Aim: The study aimed to explore maternity nurses' perception regarding challenges facing ten steps to successful breastfeeding implementation at El-Shatby University Hospital Alexandria Egypt as well as to identify their suggestions for improvement and enhance successful implementation. Methods: By using descriptive qualitative research methodology approach a focus group discussion and in-depth personal interviews were used to explore maternity nurses' perception regarding challenges and constraints to implement tens steps at El-Shatby University Hospital and also to identify their suggestions for improvement. A focus Group Discussion Questions Guide, and Interview Questionnaire were developed and utilized for data collection by the researchers. This study was conducted 6 months later after the onset of the implementation of The Baby-friendly Hospital Initiative (BFHI) first monitoring survey at El-Shatby hospital by Abul-Fadl et al,2018. Subjects: Fifty-five nurses had participated in the study; 12 nurses attended the focus group and 43 nurses had interviewed by the researchers. The nurses' responses were transcribed and coding using Nvivo software program. Thematic analysis was conducted and consensus between the researchers was reached to validate the themes. Results: Nurses had positive perception regarding ten steps of breastfeeding implementation, but they perceived a lot of barriers and challenges to implement ten steps to successful breast feeding. Five main themes were raised by the study including; Nurses related issues, Physician related issues Maternal related issues, Postpartum related issues and lastly Administrative related issues Conclusion: Various challenges in implementing the ten steps were identified and suggestions were directed to improve the staff education especially hands on education including counseling and communication technique. More health education messages especially during antenatal period were highly recommended for both new mothers and their family members as well. Intensified need for managerial support for skin to skin (STS) practice at delivery room was recommended.
\end{abstract}

Keywords: Breastfeeding, Maternity nurses, Baby-Friendly, Ten steps, University hospitals

DOI: $10.7176 / \mathrm{JHMN} / 69-06$

Publication date: December $31^{\text {st }} 2019$

\section{INTRODUCTION}

Breastfeeding is one of the most effective ways to ensure child health and survival. It is also a critical key to achieve many of the Sustainable Development Goals (SDGs) (United Nation, 2018). Promotion of breast feeding is a challenging issue and become a public health priority by the aggressive marketing of industry and its unethical marketing practice and manipulative tactics of breast milk substitute. Promotion, supporting and protecting breastfeeding has been a mandate of the global strategy of Infant and Young Child Feeding (IYCF)(WHO,2003).

This global strategy calls for making practices in maternity facilities baby friendly by implementing the ten steps of the baby-friendly hospital initiative (BFHI) which is a World Health Organization and United Nations Children's Emergency Fund UNICEF/WHO supported program. Egypt was one of flagship countries which started with BFHI program. This program has been started in 1991 and was updated in 2009 and revised in 2018 to catch up with other programs as Expanded Program of Immunization (EPI) and Control of Diarrheal Disease (CDD) program (UNICEF/WHO, 2016 \& WHO,2018b). Furthermore, the BFHI material training and advocacy was updated and revised for clarification and further interpretation of the ten steps, based on the emerging evidence. This included; emphasizing in "Step 1" the implementation of the code, and in "Step 4" the initiation of breastfeeding through immediate skin-to-skin (STS) contact for one hour or up to the first suckle. In addition, the BFHI material was further updated by categorizing the ten steps into critical management procedures and key clinical practices. The former highlighted emphasis on the Code and relevant resolutions and the need to establish ongoing monitoring and data management systems. While the latter (key clinical practices) added counseling families in addition to mothers in step 3, and managing common difficulties in step 5, and guiding mothers to recognize and respond to infant feeding cues. Step 9 was adapted to counsel mothers on the use and risks of bottles 
and pacifiers. Accordingly, the ten steps remain in context as they were written originally but with further clarification for improving its implementation (WHO \& UNICEF,2018).

Maternity nurses are the key element of breastfeeding care and they play an essential role in promoting the ten steps in hospitals (Weddig et al, 2011). Moreover, their support is essential for the optimal ten steps implementation (Edward, 2011). Through advancing education and training and by working with each other with the medical staff, maternity nurses should work hard towards achieving the aim of promoting and protecting breastfeeding through the baby friendly Hospital Initiative (BFHI).

\section{SIGNIFICANCE OF THE STUDY:}

The Baby-friendly Hospital Initiative (BFHI) is an international program. However, its implementation faces many challenges in the form of its sustainability. (UNICEF/WHO, 2016). In 2011, Schmied et al had studied the Australian health professionals' perceptions regarding implementing the baby friendly health initiative in order to protect, promote and support breastfeeding. The study's participants considered BFHI implementation a high priority and an essential set of practices that would have positive benefits for babies and mothers both locally and globally as well as for health professionals. However, its implementation faced with numerous challenges including a number of organizational constraints. The study concluded that there was a contradiction between the broad philosophical stance and best practice approach of this global strategy and the tendency for health professionals to focus on the ten steps as a set of tasks or a checklist to be accomplished (Schmied et al, 2011).

In another study Cunningham et al (2018) tried to evaluate perceptions of maternity nurses of implementing the ten steps to successful breastfeeding in an urban Texas hospital. Maternity nurses perceived a number of barriers to implementing the ten steps to successful breastfeeding including nurse staffing shortages, variations in practice among nurses, different levels of nurse education and knowledge about breastfeeding, lack of parental awareness and knowledge about breastfeeding, and postpartum issues such as maternal fatigue. Moreover, the study called for the essential need for hospital leadership support for full implementation and policy adherence.

In Egypt Fawzi et al (2013) assessed the current status of implementation of the ten steps of BFHI and the mother friendly practices in El-Shatby University Hospital. The study was carried out on a sample of 120 mothers who were interviewed from each of the service units of the obstetric antenatal, postnatal normal vaginal birth, postnatal cesarean section (C-S) deliveries and neonatal intensive care units (NICUs), The study results had found that, education about mother friendly practices was high $(87.3 \%)$ while education about early initiation of breastfeeding through early extended one hour skin to skin (STS) was lowest (0\%). Initiation of breastfeeding during the first hour was high but mostly without STS (73\%). Teaching mothers how to breastfeed was also high (73.3\%), but remaining practices were low, particularly early separation and supplementation with fluids $(30 \%)$ and milks (66.7\%). Company representative were not allowed to contact mothers in $(66.7 \%)$. NICU practices encouraged milk expression in $16.7 \%$, and mother to hold her baby STS in $23.5 \%$. The researchers concluded that overall practices were low and indicate the importance of the presence of an installed in-service training program or monitoring system to ensure sustainability of the program inputs.

Furthermore, Abul-Fadl et al, study in 2018 aimed to present and compare the monitoring activities that were carried out by 6 university hospitals in Egypt for assessing BFHI after its implementation. EL-Shatby University Hospital (El-Shatby UH) was one out of the six hospitals included in Abul-Fadl et al study (AinShams UH, AlHussein (Azhar)UH, Assiut UH, Benha UH and Cairo Kasr ElAini UH). The hospital was evaluated by using the monitoring tools of UNICEF/WHO BFHI (2009). Four monitoring visits were conducted over the period of 3 months and involved interviews with 83 mothers in maternity wards and NICUs, 90 pregnant women in antenatal clinics and 42 clinical staff in the service wards and 28 baby observations. Data were collected and analyzed quantitatively into the UNICEF/WHO BFHI summary sheets of the tools. The study results found that, El-Shatby UH met BFHI global criteria for steps 1, 2 and 3 (the mean scores\& the final percent score were 61.44 $\pm 41.32(89.5 \%), 45.85 \pm 35.12(85 \%)$ and $83.47 \pm 12.47(98.4 \%)$ respectively). On the other hand, the hospital had lower mean scores in step $4(5.275 \pm 6.416(14.3 \%)$, step 5 (26.35 \pm 18.99 (49.4\%), step 6 (10.825 $\pm 9.68(19 \%)$, step7 $(12.9 \pm 12.49(19 \%)$, step8 $(33.95 \pm 19.61(42.9 \%)$, step9 $(11.47 \pm 8.82(31.2 \%)$ and with step10 $(33.95 \pm$ 19.61(71.4\%)) (Abul-Fadl et al., 2018).

Certainly in line with the previous studies (Fawzi et al, $2013 \&$ Abul-Fadl et al., 2018), there was a need to explore and investigate what behind hindering ten steps implementation in El-Shatby University Hospital. So, more in-depth interview and interactive discussion will allow for broader and deeper insights and draw out more useful data about what maternity nurses' opinions regarding improving breastfeeding practices in maternity services which still remains a challenge.

\section{AIM OF THE STUDY:}

The aim of the study was to identify maternity nurses' perception regarding challenges facing ten steps to successful breastfeeding implementation at El-Shatby university hospital, Alexandria, Egypt. 


\section{THE RESEARCH QUESTIONS:}

This study tried to answer the following questions;

1- What are the maternity nurses' perceptions regarding ten steps to successful breastfeeding implementation at El-Shatby University hospital?

2- What are the nurses' suggestions to overcome the faced challenges?

\section{MATERIALS AND METHOD}

\subsection{Materials}

\subsubsection{Study design:}

A descriptive qualitative research methodology was conducted by using a dual step approach that included the same set of open-ended questions asked in a focus group discussion and during maternity nurses' in-depth personal interviews. Based on the Baby-friendly Hospital Initiative (BFHI) first monitoring survey results (Abul-Fadl et al,2018). It was important to explore nurses' perception regarding challenges and constraints to implement tens steps and to identify their suggestion for improvement. The in-depth focus group discussion enabled participants to explore the feelings within the group dialogue. Responses from the in-depth personal interviews allowed the researchers to check for possible variations across individuals and identify emerging themes involving in personal interview allowed also for identifying different personal experience that can't be explored during focus group. Moreover, it is worth mentioning that due to staff shortage in each work shift and for the sake of hospital clients' nursing care; in-depth personal interview was the most applicable method for meeting nurses according to their free duty time that's why 12 nurses can be collected in the focus group versus 43 nurses in the personal interview.

\subsubsection{Study setting:}

The study was carried out at El-Shatby University hospital in Alexandria, Egypt. This hospital was one of the first university hospitals to become accredited as Baby Friendly in the early 1990s when Egypt was chosen as one of the 12 flagship countries to adopt the Baby Friendly Hospital Initiative (BFHI). However, the major challenge facing BFHI as any other program is sustainability.

\subsubsection{Study subjects:}

Fifty-five maternity nurses had participated in the study, 12 nurses attended the focus group and 43 nurses had individually interviewed by the researchers.

\subsubsection{Study tool (Instrument):}

A focus Group Discussion Questions Guide, and Interview Questionnaire were developed by the researchers in Arabic language after reviewing related literature, UNICEF monitoring tools for BFHI, and previous studies (UNICEF/WHO, 2016, Cunningham et al,2018). This tool includes two parts; the $1^{\text {st }}$ part including: Nurses' personal data such as age, level of education, their working units (labor, antenatal, postpartum or recovery, family planning), years of experiences. While the $2^{\text {nd }}$ part including the remaining questions that were developed to determine nurse's perception regarding ten steps implementation and their suggestion for improvement as shown in table (1)

Table (1): Focus Group Discussion Questions Guide and Interview Questionnaire

\begin{tabular}{|c|c|}
\hline Introduction & $\begin{array}{l}\text { In this part the researchers introduce themselves (name, occupation) and explain } \\
\text { the study purposes and confirming the confidentiality of data as well as getting } \\
\text { their permission for recording their views. }\end{array}$ \\
\hline $\begin{array}{l}\text { Engagement questions } \\
\text { (ice breaking questions) }\end{array}$ & $\begin{array}{l}\text { Can you talk about you in two words, which unit you work in and one thing you } \\
\text { like to do when you are not working }(5 \mathrm{~min}) \text {. }\end{array}$ \\
\hline \multirow{7}{*}{$\begin{array}{l}\text { Exploration questions } \\
\text { (Key questions) }\end{array}$} & What do you know about hospital breastfeeding policy? How to improve this step \\
\hline & $\begin{array}{l}\text { What kind of training do you have - you received about breastfeeding (type of } \\
\text { training) do you feel it was adequate to show mothers how to breastfeed? }\end{array}$ \\
\hline & Do you think your hospital should promote breastfeeding? why or why not? \\
\hline & $\begin{array}{l}\text { Do you think that all pregnant women at the hospital be informed about the } \\
\text { benefits and management of breastfeeding? }\end{array}$ \\
\hline & $\begin{array}{l}\text { What do you think about showing mothers how to breastfeed and how to maintain } \\
\text { milk supply if they should be separated from their babies? }\end{array}$ \\
\hline & $\begin{array}{l}\text { Do you think that offering no food or drink other than breast-milk - unless } \\
\text { medically indicate is applicable? Why and why not? }\end{array}$ \\
\hline & $\begin{array}{l}\text { Many supporters of breast feeding recommended getting a newborn baby skin to } \\
\text { skin (STS) with positioning the baby on his mom's chest, within an hour after } \\
\text { birth do you feel this is important? What challenges or barriers may arise in doing } \\
\text { this for the mothers (Initiate breastfeeding with one half hour through first hour } \\
\text { skin to skin contact)? }\end{array}$ \\
\hline
\end{tabular}




\begin{tabular}{|c|c|}
\hline & What do think about Encouraging on-demand feeding and its applicability? \\
\hline & $\begin{array}{l}\text { Many breastfeeding supporters recommended teaching mothers that supplement } \\
\text { feeding should be avoided unless medically indicated. What do you think about } \\
\text { that? }\end{array}$ \\
\hline & What do you think about your hospital rooming in policy? \\
\hline & $\begin{array}{l}\text { What breastfeeding support resources for post discharge do you recommend to } \\
\text { mothers }\end{array}$ \\
\hline & What are your suggested measures to overcome these challenges for each step? \\
\hline $\begin{array}{l}\text { Exist question (closing } \\
\text { question) }\end{array}$ & Is there anything else would you like to say? \\
\hline
\end{tabular}

\subsection{Method:}

The study was conducted through the following phases;

\subsubsection{Administrative process:}

- An official letter was directed from the faculty of nursing to hospital manger and head of maternity department to obtain their approval for collecting the necessary data from the study setting

- Meetings were held with hospital manger and head of maternity department\& head nurse to clarify the purpose of the study and to gain their cooperation during data collection. (This study considers a feedback study that based on the Baby-friendly Hospital Initiative (BFHI) first monitoring survey results (Abul-Fadl et al,2018).

- After receiving the permission from the hospital manger and hospital head nurse a list of nursing staff (from all the shifts; including morning, evening and night shifts) the nurses were selected randomly according to their availability and their acceptance to participate in the study. 12 nurses were selected to participate in the focus group including the head nurse and 43 nurses had individually interviewed by the researchers.

\subsubsection{Content validity:}

- The content validity of the study instrument was reviewed by an expert jury consisting of international board-certified lactation consultants, 5 community health staff members to check the comprehensiveness of the FGDs guide, clarity, and applicability. Their suggestions and modifications were done.

\subsubsection{Pilot study (Mock):}

Mock (pilot study) was done on 5 nurses who didn't included in the study in order to ascertain the relevance, clarity and applicability of the tools, test wording of the questions and estimate the time required for filling the questionnaire. Based on the obtained results, the necessary modifications were done.

\subsubsection{Data collection:}

- Two FGDs were conducted to elicit qualitative data from the nurses using Focus Group Discussion Guide.

- The researchers started by recruiting from the above mentioned setting the nurses who wanted to be included in the Focus Group after informing them of the purpose of the study.

- A group of about 6 nurses participated in each FGD session.

- FGDs were held in comfortable and accessible place in the hospital.

- The FGDs places were arranged so that all participants can view one another using U- shaped seating, identification cards were placed in front each respondent which contained his number in the group. All FGDs were audio taped.

- The researchers started the FGD by introducing themselves, and the objectives of the sessions were clarified to the participants in order to build rapport with participants.

- After that the researchers introduced ice breaking questions, followed by Exploration Questions; using verbal and nonverbal communication such as head shaking, and asking open ended questions in accordance to FGDs guide.

- The moderator (researchers) actively encouraged participation of everyone in the group. An important job of the moderator was to solicit input from all group members and not leave few vocal respondents to dominate the discussion.

- The need and importance of every respondent's input and opinion were emphasized by the moderator.

- Diversity of comments and opinions among the group was encouraged and flexibility for clarification and probing were allowed.

- The Focus Group sessions were recorded using record tape in addition to handwritten field notes. The session was audio recorded so that responses could be transcribed and prepared to analysis step. Moreover, the participants' answers were restated back to them for member checks, and the two coders coded the data and discussed themes until consensus was reached.

- Each Focus Group took approximately from one hour and half to two hours.

- The moderator ended the focus by Exit questions (closing question) and thanked the respondents for their 
contribution and cooperation.

- After each session the researchers examined the information collected by matching between the tape and the notes taken in order to overcome and fill any missing data.

- Additionally, data was collected by in-depth personal interviews across the three-working shift. Each personal interview took approximately from one hour to one hour \& half. Data was collected during May 2019.

2.2.5 Data analysis:

Recording of the focus group were transcript and checked for data accuracy and quality. The focus group was independently coded by the researchers using qualitative themes analysis technique and Nvivio software. The common themes and subthemes were developed and validated via group discussion and in-depth interview.

\subsubsection{Ethical considerations}

- Informed written consent was obtained from all participants after providing an appropriate explanation about the purpose of the study and nature of the research.

- The confidentiality and anonymity of individual responses, volunteer participation and right to refuse participating in the study were emphasized to the participants.

\section{RESULTS}

Fifty five nurses had participated in the study, 12 nurses attended the focus group and 43 nurses had interviewed by the researchers, the mean age of the participants was 35.6 with the age ranging from 21 to 56 years old, the average years of experience at maternity or child care was 13 years $(\mathrm{SD}=13.1)$, and $70 \%$ of nurses were married.

Most of nurses reported positive perception regarding the importance of ten steps of breastfeeding implementation and they reported different barriers facing its implementation. The subjects' response themes classified; as nursing related issues, physician related issues, maternal related issues, post partum related issues and administrative related issue (Figure 1).

Theme I: Nursing related issues:

This theme covers six subthemes: (1) nurse staffing shortage and over workload, (2) lack of time and work practice priority, (3) nurse's resistance to informal peer training, (4) nurses' education and breastfeeding related knowledge, (5) lack of communication and counseling skills and (6) lack of continuity of care.

\section{Nurse staffing shortage and over workload:}

Most of nurses had reported nursing staff shortage especially in evening and night shifts at all wards including recovery rooms as the main barrier to implement ten steps. "Imagine there are only two nurses in the afternoon shift for this entire ward, at some days we are 2 nurses for 35 clients and more". "It is difficult for me to give this health education for every mother how I can do this am alone in this ward."

Lack of time and work practice priority:

Four nurses had stated that the amount of time required to give health education to mothers is not enough, as they have no time to offer breast feedings messages. "We have no time to teach mothers, I have another responsibility, provision of medications to mothers is my top priority and this is my main responsibility". "There is no time to provide complete health teaching" "I will be punished for not giving the mothers' medication or for any errors, but I will not be punished if I forget to talk about breast feeding"

\section{Resistance to informal peer training:}

Resistance to change and gaining training is raised by some nurses. Professional and knowledgeable nurses faced with a lot of resistance in provision informal training about breast feeding to their elder nurses' colleagues at the same unit. Nurses added "It is difficult to orient or train elder nurses especially who resist change by newly professional graduated ones"

Nurses' education and breastfeeding related knowledge:

Variation in nurses' level of education (higher education, associate and secondary education level) is linked also to variation in their breastfeeding related knowledge and practice. Five nurses with secondary education stated that, their education not stressed on breastfeeding as a topic, while others depending on their own breastfeeding experience "We did not receive any knowledge regarding techniques of breast feeding before and also we did not hear about breast feeding policy". While other nurses $(n=6)$ who had maintained their knowledge with breastfeeding courses called for hands on job training especially the practical part. "I received lectures before for breast feeding and attained several courses, but we still need practical experience with updates as skin to skin position".

Lack of communication and counseling skills:

Some nurses talked about the importance of receiving training on practicing counseling and effective communication skills with the mothers and their relative. Nurses raised another important theme related to their pitfalls in communication and counseling pattern. One nurse stated that "I think you can get the knowledge anywhere but the most important is putting the self in a real practical situation to counsel and communicate with the mothers in presence of their babies, we need more practice". Moreover, gaining the mothers' feedback about 
breast feeding related knowledge is neglected by majority of nurses and this considering another barrier to implement ten steps. "Really; most of us when talking about breast feeding, we did not gain the mothers' feedback, so we did not know if they really understand well or not."

\section{Lack of continuity of care:}

Another barrier and constraints that faces breast feeding ten steps implementation is lack of consistency in clinical practice as a result of absence of hospital breast feeding guideline for both physicians and nurses. "Normal delivered mums just stayed one day, so you can't judge their practice. However, we told them to come back to the breastfeeding clinic if there is any question, I think no one comes. They came only to take milk substitute".

Other nurses stated that lack of continuity of care is related to the self believe in breastfeeding messages which differ from nurse to nurse and from shift to shift with a different nurses' responsibility from labor and delivery to postpartum care area "When I received the mother from the delivery room or labor room really I did not asked if any person talked with her about breast feeding or not." I suppose that mothers who receive antenatal care know all things about breast feeding",

Lack of stability in giving complete health education messages to mothers is another barrier facing the ten steps, in line with that, some nurses added "really some points when providing health education are missing from our messages such as cup feeding, how to express breast milk" milk expression"

\section{Theme II: Physician related issues:}

This theme covers three subthemes including: (1) Violation of breastfeeding code of marketing, (2) Fear of applying skin to skin (false believes regarding STS) and (3) Role conflict between pediatrician and obstetrician.

\section{Violation of breastfeeding code of marketing:}

Physicians were blamed for suggesting supplementation to mothers, which may contradicting with the nurses advice "You told the mother try to give your breast milk for the $1^{S T} 6$ months, on the other hand the physician giving the mother or the father a paper with supplementation for her baby". Moreover, one nurse stated that, "I can't talk about formula side effects because formula sometimes is the correct option for the mother and baby and in some cases physicians themselves recommend it to another mothers"

Fear of applying skin to skin (false believes regarding STS):

Some nurses criticizing physician fear of conducting STS especially pediatrician as they stated that "most of pediatrician believes that skin to skin interfere with the baby routine assessment and may exposed babies to distress and hypothermia. Another nurse added "For the purpose of making the newborn routine investigation immediately after birth, pediatric physicians refused skin to skin and rooming in; this point contradicting with the ten steps".

Role conflict between pediatrician and obstetrician:

Some nurses discuss the issue by wondering who should give them instructions and orders, since some of them were affiliated to the El-Shatby Pediatric hospital managers and other are affiliated to El-Shatby Maternity hospital managers, so they faced role conflicts between both authorities. "We are as nurses follow orders from our manager. That's mean nurses who working at maternity departments follow orders of maternity managers, while those working at pediatric departments follow orders of pediatric managers. So that if these mangers didn't support STS as an example, we can't do it'"

\section{Theme III: Maternal related issues:}

The maternal related issues can be classified into three subthemes: (1) maternal culture, (2) maternal previous experience and preference, and (3) maternal awareness regarding breastfeeding.

\section{Maternal Cultural:}

Culture is a barrier in some communities to breast feedings, some incorrect information and practice in certain communities may contradict with the ten steps. In this regard the studied nurses told that, "One mother from Borg El-Arab "Arabian" told me before that people there give the baby some herbs for several days after delivery to clean up his stomach after delivery". Another nurse said that "The mother in-law plays a dangerous role either to support breast feeding or not; some grandmothers, can wait they introduce foods to babies by their $2^{\text {nd }}$ month" "I know mothers giving baby rest (medication to relive baby colic) to their babies instead of one breast feeding"

\section{Maternal previous experiences and preference:}

From nurse's perspective, the mother previous experience plays a role in choosing either to breast feed their babies or not. Nurses added "Mothers have the right to choose how to feed her child. But of course, after receiving breastfeeding correct information". One nurse added "The mother with previous bad experience as she had failed in feeding before may think that she can't breast fed again. The problem her that this mother may guide other mothers and may give wrong information which spread infectiously among newly mothers with no previous experience". Two nurses stated that, mothers' working is another challenge to implement this steps "Some working mothers after delivery asking me to write down any formula for their babies they prefer to give formula when they are at work, they did not know how to express and storage their milk"

Maternal awareness regarding breast feeding:

Poor maternal awareness was referred as a serious barrier for ten steps implementation, nurses stated that some mothers, has misinformation about colostrums. "Mothers think that there is no milk at the first days after delivery; 
that is why they believe in giving any formula" "Mothers also unaware of the importance of skin to skin and rooming in". Another nurse added "Mothers with twins think that she can't breast fed their children effectively and she called for formula feeding". "In order to make their first days after delivery tolerable, mothers behave in a wrong manner and may take wrong decision to use bottle feeding and use pacifiers". Antenatal breastfeeding education for pregnant women focusing on their knowledge feedback both to correct their misconception and to raise their awareness is recommended by nurses as an effective option to implement ten steps effectively.

\section{Theme IV: Postpartum related issues:}

Postpartum issues were classified into five subthemes: (1) Maternal postpartum status, (2) Routine, postpartum care, (3) Family member support, (4) Privacy, and (5) Infant or newborn security.

\section{Maternal postpartum status:}

Maternal postpartum condition is affecting their ability as well as decision to breastfeed their babies, so it may hinder breastfeeding practice. Some nurses stated that "This new generation of new mothers are very weak, they hardly tolerate to breast fed their babies especially in the first hours after delivery, they have no power to struggle", while some nurses perceived that mothers with Caesarean Section are very fatigue and exhausted to fed their babies. "with a high rate of surgical deliveries, the mother' ability to listen and communicate with the nurse may be corrupted because of their fatigue and they lack the patience to listen". Some nurses stated that "Presence of any complications for the mothers and newborn are other barriers to immediate skin to skin contact and early initiation of breast feeding"

\section{Routine postpartum care:}

Some nurses stated that some routine postpartum procedures for both mothers and babies may delay early initiation of breast feeding as they reported that "In the delivery unit, the baby is transferred to normal babies' room to stabilize his condition first and to make certain investigation. This may take about 3 to 4 hours then transferred to his mother or his family outside the unit". One nurse added, "By priority most of us were occupied by doing post partum care to mothers, making vital signs and giving medications rather than talking with her about breast feeding"

\section{Family members support:}

Most of nurses believe that presence of family members' support helps in improving breastfeeding practices and support the achievement of ten steps of successful breastfeeding. They added with happy faces (smiles) that, "Family support will decrease our workload as well as will help mothers to be well oriented and informed on breastfeeding.". However, some nurses see it is important to accompany only one person per each mother immediately after labor to help her but it is forbidden by the hospital in delivery unit, they stated that, "Presence of family support will help mother to carry her baby and help her move easily after delivery. But it is difficult to be achieved because of the hospital policy not allowed the mothers to accompany any relative especially in the normal delivery department."

Lack of privacy:

Family members including relatives and visitors especially at the post partum wards were commonly an obstacle to ten steps implementation, nurses stated that "Mothers lacking their privacy when their room is crowded with visitors, that's why they may delay feeding their babies". Some mothers were afraid to feed their babies in front others as they believe in evil eye "Some mothers delay breastfeeding especially in front her husband relatives even if they are women".

Lack of newborn safety and security:

Lack of newborn safety and security was another barrier for applying rooming in. This issue was raised by nursing staff as fearing from stealing babies from their mothers especially in the recovery and post partum room. Nurses stated that "No relative permitted to be available beside mothers. There was previous history "Some babies were stolen from their mothers before and the mothers forced to be separated from their babies and the newborn can be discharged to his father or relative. We have no Rooming in here" Nurses added "This is our responsibility if any baby was lost, we are subjected by court"

Theme V: Administration related issues:

Administrative related issues cover four important subthemes: (1) Breastfeeding policy, (2) Discharge form, (3) lack of accountability and continuous monitoring, and (4) Breastfeeding protocol.

Breastfeeding policy:

Although, breastfeeding policy is one of the parameters for successful implementation of BFHI activities, the studied nurses stated that there is poor orientation about breastfeeding policy application details "factually, before receiving our training not all of us was aware by this policy or know its elements, and we are not aware why it is important".

Discharge form:

Discharge form is a card form provided for the mothers at the discharge time, it contains a written instruction to be followed at home. The studied nurses confirmed that even though the availability of discharge form, its content instruction didn't comply with the goal of breastfeeding sustainability. One nurse stated, "The discharge form of 
the hospital itself contradicts ten steps implementation" "The discharge form should be modified to omit on schedule breastfeeding as it written as the first instruction in the discharge form." Another nurse added "Before receiving our training we were telling the mother to breast fed her baby every 2 hours and it was wrong to say that"

Lack of accountability and continuous monitoring:

The key facilitator for sustaining the breastfeeding is strict accountability to do. The studied nurses declared that, "The fact of being responsible for what you do is pushing us to work. In fact, some nurses didn't comply with their work if they didn't know that there is a head who observe them, or continuously monitoring and evaluating them" Breastfeeding protocol:

Breastfeeding protocol serve only as a guideline for the care of breastfeeding mothers and their infants and do not delineate an exclusive course of treatment or serve as standards of medical care. Variations in treatment may be appropriate according to the needs on individual base. The studied nurse stated that they didn't have a written protocol for breastfeeding; they appreciate the availability of such protocol in order to provide an effective breastfeeding. Some nurses elaborate on the importance of breastfeeding protocol "from my point of view, breastfeeding is important. This protocol will save our time and also make all nurses responsible and give them the sense of importance and caring provided for the breastfeeding issue". One nurse stated presence of breast feeding protocol should be a part of the hospital quality committee it is important to have written protocol and breast feeding committee at the hospital it can be linked to the hospital quality committee as the infection control committee)

Figure (1) Nursess Perregtion for the Challenges Faring Ten Steps of Suregssful Brreastfeading Implementation

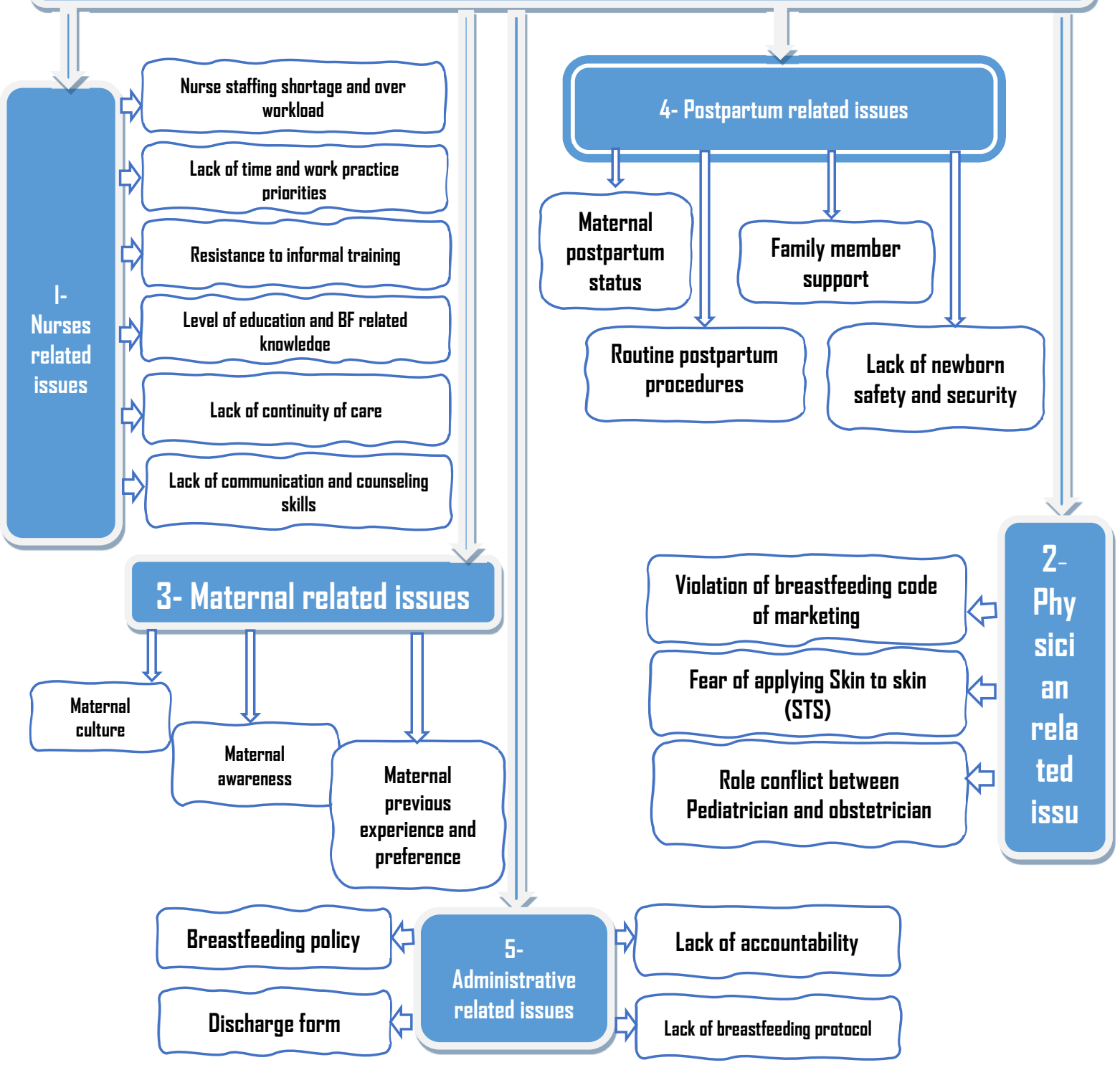




\section{DISCUSSION}

El-Shatby University Hospital is the preferred option for women in Alexandria Governorate and around provinces (Alexandria University Hospitals, 2013). It provides the high-quality services and specialized care, outstanding quality at appropriate and low prices. It also acts to achieve UNICEF's partnership with the Ministry of Health and Population flagship programs including the Expanded Program on Immunization, the Baby Friendly Hospital Initiative (BFHI), polio eradication, maternal and neonatal tetanus elimination, and the integrated management of neonatal and childhood illness (UNICEF, 2015). It also aims to achieve the sustainable developmental goals regarding reducing the neonatal mortality by 20 percent and child malnutrition by 30 percent in the targeted disadvantaged areas, where one of the six global nutrition targets for 2025 is to increase the rate of breastfeeding in the first 6 months up to at least 50\% and (UNICEF/WHO, 2016).

The Ten steps to successful breastfeeding should be integrated into maternity care to increase the likelihood that a woman will initiate and sustain breastfeeding and achieve her personal breastfeeding goals (The American College of Obstetricians and Gynecologists, 2016). The sustainability of breast feeding can be achieved mainly by those who acknowledge the proper implementation of the ten steps of successful breastfeeding and believe on its importance, so that the current study aimed to assess the maternity nurses' perception regarding challenges facing ten steps to successful breastfeeding implementation.

Since the researchers of the current study conduct in-depth interview and guided focus group discussion with 55 maternity nurses working at El-Shatby University Hospital and found that their response themes regarding the challenges facing ten steps to successful breastfeeding implementation divided into five main themes; nurses related issues, physician related issues, maternal related issues, postpartum related issues and administrative related issues.

Firstly, the current study admits that, the studied nurses had positive perception regarding ten steps of breastfeeding implementation. But they added that they are facing a lot of challenges that hinder achieving proper implementation. Nearly the same findings were reported by Daniels and Jackson (2011) since they found that, the majority of the staff had positive perceptions of the BFHI and could see the importance and benefits it holds for the mother, baby and staff additionally, the majority of the staff agreed that the benefits of breastfeeding outweighed any difficulties. Also, the same findings reported by JACOBS (2008) who found that the majority of the nurses had positive attitudes toward BFHI principles and practices.

\section{Nurses related issues}

The first theme regarding the challenges facing the implementation of ten steps of successful breastfeeding as perceived by the studied nurses in the current study was nurses' related issues. In that issue, maternity nurses discussed six subthemes.

One of the important challenges facing the implementation of ten steps of successful breastfeeding is nurse staffing shortage and over workload. In fact, nursing staff who working at the university hospitals were overwhelmed, they are responsible to do a lot of tasks either nursing related task or non-nursing related task. Staff shortage is the weapon that leads to retracting in the nurse's performance. One of the main maternity nurse's roles is to maintain mothers and their baby's safety which in directly affecting breastfeeding process especially, when they provide high priority for other procedures like preparation for diagnostic procedures, administering medication, infection control, charting and documentation and other procedures. These findings were upholding by the works of the study done by Daniels and Jackson (2011) and Wieczorek et al (2015) as they added that the BFHI poses extra work for nurses.

The second nurses' related issue as reported by the studied nurses in the current study is lack of time and work practice priorities. The benchmark to nurse-patient staff ratios is highly important parameter to maintaining and promoting better care, in most cases, it's the law. In this regards, the American Nurses Association (ANA) declared that, nurses are empowered to create staffing plans specific to each unit, allows hospitals to establish staffing levels that are flexible and account for changes including the intensity of patients' needs, the number of admissions, discharges and transfers during a shift, level of experience of nursing staff, layout of the unit, and availability of resources, such as ancillary staff and technology. (Lippincott Nursing Education, 2016).

Factually, shortage of staff put extra cases who must be cared by the same nurse, in this situation the nurse didn't fined solution to this situation except to re-planning for the shift time and implement only the essential activities. That is why; ten steps of successful breast feeding in this story may become later in prioritizing the nurse's role. In this regards Taylor et al (2011) declared that, the staff felt they were struggling to implement BFHI because the physical environment and lack of resources and staff time constrained them.

On-the-job training, also known as OJT, is a hands-on method of teaching the skills, knowledge, and competencies needed for employees to perform a specific job within the workplace. Employees learn in an environment where they will need to practice the knowledge and skills obtained during their training. OJT is one of the tools that manages the problem of staffing shortage, since they are received their needed training at their working place without interruption of schedule or working plan (Nader and Shahroz, 2011).

Although the benefits of OJT some nurses resisting the informal training, this the third nurses related issue 
discussed in the current study. In fact, these findings are clearly observed among older nurses who didn't accept to receive training by younger one. Nearly the same findings were reported by Daniels and Jackson (2011) as they reported that resistance and reluctance to change behavior are prominent in their study for "Knowledge, attitudes and practices of nursing staff regarding the Baby-Friendly Hospital Initiative in non-accredited obstetric units in Cape Town".

Furthermore, training and education on mother-friendly care practices should be conducted to equip all nurses with skills and information to improve quality mother-friendly care, and each nurse should receive an orientation to the code of marketing for breast milk substitutes (BMS) and how to intensify the ten steps of successful breastfeeding effectively (Mgolozeli et al, 2019). The same findings reported by JACOBS (2008) who found that lack of properly skilled nursing staff is a major obstacle to proper breastfeeding and infant and young child feeding management. Attention must be given to training on the more advance skills such as breast milk hand expression.

Level of education and breastfeeding related knowledge as one of the nurses related issues that discussed by the studied nurses in the current study revealed that the variation in nurses' level of education (higher education, associate and secondary education level) is linked also to variation in their breast feeding related knowledge and practice. This finding goes in line with Yang et al (2019) who looking for "Expectations and experiences of nursing students in supporting new mothers to breastfeed: A descriptive qualitative study" and found that there is evidence that theoretical and clinical education improves breastfeeding knowledge and attitudes among nursing and other health professional students prior to clinical placement, to support women who are breastfeed. Additionally, Smith et al (2012) added that barriers to key Baby-Friendly steps were overcome through creative approaches and strategic education for staff, physicians, and parents. Additionally, Cunningham et al, 2018 added that maternity nurses desired more education about breastfeeding; specifically, a hands-on approach, rather than formal classroom instruction, to be able to promote successful implementation of the Ten Steps.

Lack of nurse's education sometime assert why poor planning for nurse's work including conducting breastfeeding related care and integrating it as one of the standards of the given care. In this regard the current study revealed that some nurses perceived that poor breastfeeding implementation is due to lack of continuity of care since they have lack of consistency in clinical practice as a result of absence of hospital breastfeeding guideline for both physicians and nurses. In addition, it is related to the self believe in breastfeeding messages which differ from nurse to nurse. Indeed, poor guidance in addition to poor breastfeeding knowledge, lack of time and shortage of staffing are interrelated factors leads to lack of communication and counseling skills.

Counseling a woman about breastfeeding is one of the most important things that affecting breastfeeding sustainability. Counseling means tailor information for each case separately. This role must be conducted and it is the better chance to conduct counseling session while the mother at the hospital since they may have a lot of inquires moreover, it simplifies mother orientation regarding the presence of breastfeeding support group either inside or outside the hospital. This finding is supporting Genetu et al (2017) findings. Additionally, Cunningham et al, 2018 stated that nurses in their study perceived a number of barriers to implementing the Ten Steps to Successful Breastfeeding including different levels of nurse education and knowledge about breastfeeding, lack of parental awareness and knowledge about breastfeeding.

Counseling is not an easy procedure. It needs a well-informed nurse who equipped and prepared to meet mothers inquire related to breastfeeding and able to answer all questions or conduct proper referral at the suitable time. Sometime the maternity nurse wasn't prepared to do such counseling and provide only breastfeeding education. So, it is important to prepare them to do so by giving emphasis to the newly hired one since counseling topics differ according to the mother's needs assessment findings.

\section{Physician related issues}

The international code of marketing of breast milk substitutes is one of the important issues affecting ten steps of successful breastfeeding implementation. Where, the primary objective of the international code is to protect infant health and development through promotion, protection and support of breastfeeding. It aims to achieve this primary objective by prohibiting any form of promotional activities related to products under its scope (WHO, 2018a).

It was sorrowfully to found that, the international code of marketing of breast milk substitutes violated by those who should apply and protect it. Since the current study revealed that violation of the code of marketing of breast milk substitutes is considered as one of the important physician related issues. That shed the light on the importance to act to compelling and support all form of activities that aimed to stop such violation.

These findings support Fawzi et al (2013) who conduct a study on "Challenges Facing the "Updated Baby Friendly Hospital" Program in a Maternity Hospital in Alexandria" reported that around one third of the studied mothers were exposed to an infant milk formula company representative who offered them with gifts or free samples. In the same line, Liu et al (2014) who studied the implementation of international code of marketing breast-milk substitutes in China and found that two fifths of the mothers reported that they receive free formula samples. In addition, Smith et al, (2012) added that if a mother or infant was having trouble breastfeeding, staff and physicians would suggest supplementing with formula until breastfeeding was established. Also, the hospital distributed diaper bags at discharge that included formula samples, thereby promoting the use of formula. Some 
nurses promoted supplementation with formula when the infant was not voiding adequately, had a weight loss greater than $10 \%$, or displayed symptoms of hypoglycemia.

In this regard, the reason behind violation of the code was described by the WHO and UNICEF Joint report (2018) where, distributors of breast-milk substitutes have often been found to violate the Code by providing free or subsidized supplies to facilities or governments, and/or providing promotional materials to health facilities or health professionals. Facilities often find it difficult to resist these offers in the face of tight operating budgets (WHO/UNICEF, 2018).

Early and uninterrupted skin-to-skin contact between mothers and infants should be facilitated and encouraged as soon as possible after birth (Brimdyr et al, 2018). According to UNICEF United Kingdom (2019) "There is a growing body of evidence that skin-to-skin contact after the birth helps babies and their mothers in many ways. Calms and relaxes both mother and baby, regulates the baby's heart rate and breathing, helping them to better adapt to life outside the womb, stimulates digestion and an interest in feeding, regulates temperature, enables colonization of the baby's skin with the mother's friendly bacteria, thus providing protection against infection, stimulates the release of hormones to support breastfeeding and mothering.

According to UNICEF (2018) the first few hours and days of a newborn's life are a critical window for establishing lactation and providing mothers with the support they need to breastfeed successfully. Skin to skin (STS) is a first gift given for babies in their new world. Although STS is one of the main pillars for successful breastfeeding, the current study shed the light on the dark side of physician's/pediatrician's fear of implementing STS which is one of the physician barriers as perceived by the studied nurses.

These findings may be attributed to physician's fear from the rapid shift in baby's condition, especially among those who defined as high risk mother's babies. Since the majority of mother's who attend to El-Shatby hospital is mainly suffered from high risk condition. That's why the pediatrician felt such fear. Additionally, there is no accurate documentation for mother's history, so they decide to do STS only with those who expected to be well babies. This idea must be changed, and they must be trained to understand the major STS benefits according to the evidence-based practices and different studies declare. A scientific mind-based discussion with the pediatrician is the right solution for such barrier.

Who is responsible to implement ten steps of successful breastfeeding? Research confirms that, physicians who work closely with women and children either family practitioners, obstetricians/gynecologists (OB/GYN's), and or pediatricians play a pivotal role in increasing breastfeeding initiation and duration rates (Stolzer and Hossain, 2014).

In fact, every member of the health care team has a significant role in implementing ten steps of successful breastfeeding. Whereas, sometime role conflict between pediatrician and obstetrician regarding ten steps of successful breastfeeding was present, that was reported as one of the studied nurses' perceived challenges related to the physician. A review of the literature confirms the association between breastfeeding and improved women and babies' condition during postpartum period that exceed all the expectation as it protects babies and their mother' life as well. No doubt that, breastfeeding actually reduces maternal and neonatal and infant mortality, all of healthcare provider definitely know this fact. So, why there is a role conflict; this conflict may be attributed to the health care delivery system, that didn't clearly define such roles. Furthermore, there is no clear guidelines, no definite protocols present associated with poor policy orientation.

\section{Maternal related issues}

Not all the challenges that undermine the implementation of ten steps of successful breastfeeding related to the health team or the healthcare institution; some of these challenges are related to others as mother, family, and friends or even the support group. In this regard, the current study revealed that breastfeeding practice may be affected by maternal culture as perceived by the studied nurses. These findings shed the light on the importance of proper handling for mother's culture related to breastfeeding which may hinder the ten steps implementation, since Breast Milk Substitute (BMS) may be requested by the mother's herself or on behave their family members.

These findings support Komara et al (2007) and Cunningham et al (2018) as they reported that there are some cultures that endorse formula feeding shortly after birth. Moreover, Smith et al (2018) declared that peer counseling may be less effective in preventing premature weaning in countries where breastfeeding is not the cultural norm, where commercial milk formula is affordable and pervasively marketed. Additionally, Smith et al (2012) added that the specific barriers to step (6) verified when mothers were allowed to supplement on request since mothers may want to supplement because they desire to sleep during the night or perceive their infant is not receiving enough nutrition which considered culture theme also.

Furthermore, mother's experience of breastfeeding plays an important role in boosting the application of ten steps of successful breastfeeding. In this respect the studied nurses in the current study declared that, mothers' previous experience is another important challenge, they also added that this experience affecting breastfeeding practice in the way that rising alarm, since this experience not linked only to the mother herself but also to all nearby member that's called infectious according to the studied nurses expression. Some of these experiences come from the mother herself or from the mother's family, friends, peer, even neighborhood experience affect the 
mother. These findings encouraged the maternity nurse and breastfeeding team to build up on the positive experiences and try to find solution to the negative one. Proper planning is the base for proper implementation.

These findings go in line with Buontempo et al (2015) who stated that, mother's experience of breastfeeding is closely linked to cultural and religious background and to life experiences. Individual factors such as painful breasts and nipples and a perception of insufficient milk can affect the decision to stop.

There is no doubt that mother's experience of breastfeeding and their level of awareness are interchangeable factors. Mothers' breastfeeding awareness was also documented as a big challenge against the implementation of the ten steps of successful breastfeeding in the current study. This is considered as one of the important themes that clarified by the studied nurses. Although, the huge effort directed to increase the public awareness regarding breastfeeding, it seems to be less and the baby friendly hospitals still in need for endorsement of the breastfeeding awareness.

Such findings may be attributed to lack of breastfeeding education session during antenatal visits, especially among those who didn't comply with antenatal visits schedule. Furthermore, the deeply rooted false belief regarding that the benefit of formula milk is outweigh the breast milk which confirmed by BMS advertisements, all of these factors affecting mother decision and awareness. Therefore, healthcare providers should provide breastfeeding education to all women during their antenatal follow-up visits, especially women with low educational qualifications and no past breastfeeding experience. Breastfeeding awareness session must be a part of routine care provided at any maternity or pediatric healthcare institution either at the primary or secondary level of care.

These findings were documented in a study done at the U.A.E.(2018) entitled "Knowledge, attitudes, and practices of breastfeeding among women visiting primary healthcare clinics on the island of Abu Dhabi, United Arab Emirates" where the findings of this study confirmed also that, although breastfeeding knowledge in the U.A.E. was generally good, breastfeeding practice being suboptimal. Modifiable factors that was found to predict exclusive breastfeeding includes breastfeeding knowledge and mothers' employment status. The most common reasons for stopping breastfeeding as perceived by the studied women were; their perception regarding insufficient breast milk production and their babies seeming unsatisfied or hungry after feeding (Al Ketbi et al, 2018).

\section{Postpartum related issues}

Another important theme in the current study was stated by the studied nurses was postpartum related issues that cover maternal postpartum status, routine postpartum care, family member support, privacy, and infant or newborn security.

Maternal postpartum condition was reported by the studied nurses in the current study as one of the themes affecting the implementation of ten steps of successful breastfeeding. Women postpartum condition such as after pain, fatigue, exhaustion, fear from site of surgery in case of C.S, post anesthesia condition, needs for assistance and help and mother psychological condition are factors affecting mother acceptance, active and early initiation of breastfeeding and skin to skin.

These findings agreed with the results of a study entitled "Maternity Nurses' Perceptions of Implementation of the Ten Steps to Successful Breastfeeding" which revealed that the studied nurses perceived a number of barriers to implementing the Ten Steps to Successful Breastfeeding including postpartum issues such as maternal fatigue during recovery interfered with skin-to-skin positioning (Cunningham et al, 2018).

Additionally, the current study shed the light on important issue regarding the maternity nurse and routine postpartum procedures. Since, the maternity nurse who responsible to provide postpartum care did not focus on breastfeeding issue especially when they are concerned with dealing with mother's condition, postpartum hygiene, observing sign of bleeding and administering medication. These findings may be attributed to that the maternity nurses may be planned to put priorities to the life-saving procedures of the mothers and did not care for breastfeeding, they also did not accountable for delaying or negligence of it. Also, there is no clear description of nurse's role regarding breastfeeding as well as no guideline available. In addition to the role conflict especially with mother-infant separation (mothers were cared by maternity nurses and their babies cared by pediatric or NICU nurses), so who is responsible for breastfeeding. The same findings agreed with the results of a study done by Cunningham et al (2018) who revealed that the studied nurses perceived that routine required procedures during recovery care affecting breastfeeding practice.

Everyone plays an important role in protecting, promoting, and supporting breastfeeding involves family, friends and even society as a whole. There is no doubt that, breastfeeding is more successful when family members are involved. A recent research in Yogyakarta concluded that, partners and husbands are the biggest influencer in breastfeeding success (Ratnasari et al, 2017). Grandparents also play an important supporting role, especially grandmothers or mothers in law. Some things partners and family members can do such as give the new mom praise and encouragement for how hard do. Offer to help with baby or child-care, household duties, and take the baby out for a walk to let new mom sleep. All of these forms of support are crucial especially with those who did not have previous experience with breastfeeding before.

The studied nurses in the current study perceived that family member support helps in improving 
breastfeeding practices and support the achievement of ten steps of successful breastfeeding. Also, they added that, family support will decrease workload as well as helps them to be well oriented and informed on breastfeeding and give them extra chance to find answers for their inquires that they may have. Positive family support is important, and the negative one must be handled.

The current study finding goes in line with Kavle et al (2019) who declared that engaging community leadership in BFHI is important in revitalization of the BFHI. Moreover, Pérez-Escamilla et al (2016) added that community support is a key for sustaining the short-term breastfeeding benefits obtained from the BFHI steps requiring implementation only at the maternity ward. Avoiding in hospital supplementation appears to be another key step for breastfeeding success, perhaps reflecting adequate implementation of the rest of Ten Steps.

In contrast, WHO/UNICEF (2018) declared that breastfeeding support groups has proven very difficult to implement for most facilities providing maternity and newborn services, since many of these do not have sufficient staff to work outside of their own facility. In most settings, maternity and newborn facilities have not been held accountable for outreach into the community. So that Smith et al (2018) focus on the importance of community mobilization and stated that it was identified as key interventions for promoting optimal breastfeeding practices. Interventions that include peer counseling are more effective than those without peer counseling.

Most of the nurses in the current study perceived in the fact that lack of privacy affecting mother's decision to breast feed their babies. These findings may be resulting from poor hospital design that's didn't maintain mother's privacy during breastfeeding, where there is no special places available for breastfeeding and the problem appears difficult with high number of mothers and their visitors at the same room. Moreover, visitors may didn't respect women privacy and they may interfere negatively with the practice as they may give negative comments or even pass their negative experience and attitude to the mothers. Sometime lack of privacy that may be combined with the fear of evil eye may affect breastfeeding. Additionally, for religious reasons, the mothers cannot breastfeed their babies in front men.

The same finding was reported by Taylor et al (2011) as they studied "Staff Perceptions and Experiences of Implementing the Baby Friendly Initiative in Neonatal Intensive Care Units in Australia" found that privacy is an important issue affecting breastfeeding practices.

Security issue is one of the studied nurses perception regarding factors affecting successful implementation of breastfeeding, lack of newborn's safety and security was an alarming issue that's actually very difficult to managed from the nurses point of view, since they afraid to be subjected by court if they lose any baby they cared for. From the legal perspectives, nurses are responsible to protect both mothers and their babies as well and they are responsible to maintain their safety. The issue of baby separation comes due to security reason as administrative intervention to protect babies from thieves. To sum up, rooming in is highly important, and for sure the security of the newborn is mandatory. The hospital administrators can protect their patients if they put a proper plan for this. Sometime the legal action such as penalty must be become sharp and media must be interfered to orient the public about such penalties. Moreover, a special thank and recognition must be considered to the role model hospitals that can fight all these challenges and become designated baby friendly hospitals. Nearly the same findings reported by Turner-Maffei and Cadwell (2004) as they added that special recognition to the designated baby friendly hospitals must be encouraged.

In this regard, Taylor et al (2011) added that the physical condition of the infants or their prematurity often precluded them from feeding directly from the breast and may leads to separation from their mothers. Importantly, the physically separated hospital spaces of postnatal care and NICU meant that mother and infant were separated. They were treated as separate beings rather than a dyad and the interdependence of their relationship was difficult to maintain. The pediatrician stated, "The mother and baby aren't seen as being inseparable; they're seen as being separate and treated separately."

\section{Administrative related issues}

The last but not the least important theme in the current study as reported by the studied nurses was related to administrative related issues. There is no doubt, that presence of clear Policies that protect the right of a woman and her child to breastfeed in public and that accommodate milk expression, such as insurance coverage for breast pumps, paid maternity leave, on-site childcare, break time for expressing milk, and a clean, private location for expressing milk, are essential to sustaining breastfeeding (The American College of Obstetricians and Gynecologists, 2016). The discussion on policy related issues take us to new theme in the current study which highlight the administrative role in enhancing breastfeeding sustainability in the form of administrative related issues.

In this regard, the studied nurses in the current study shed the light on the policy related issues. Since they perceived that policies that was not clear to the most of them and thereby, didn't clear to all health care team as they perceived. In the same context a study done in Saudi Arabia (2017) by Al-Madani and Abu-Salem who studied "Health professionals' perspectives on breastfeeding support practices" and documented that there were no breastfeeding supporting policies followed at the studied hospital. Also, Weddig (2011) added that the studied nurses concluded that hospital polices affecting BF practices. 
Although, monitoring for the implementation of ten steps of breastfeeding start by looking for policies as the first step done by hospital who acts to be baby friendly hospital designated, no doubt that, El-Shatby University hospital's administrators are well oriented this point. But why there is reluctance in its implementation for such step? Are they satisfied that they distribute breast feeding policies as material only to be designated?

These findings may be confirmed by the fact that reported by WHO and UNICEF joint report (2018) entitled "Implementation guidance: Protecting, promoting and supporting breastfeeding in facilities providing maternity and newborn services: the revised baby-friendly hospital initiative" which revealed that many facilities appear to make changes in their policies and procedures to obtain the "Baby-friendly" designation, but then drift back into old ways over time, especially when there are no regular monitoring systems in place.

To sum up, lack of clear breastfeeding policy in turn leads to role ambiguity and lack of accountability. This is the second theme related to administrative related issues reported by the studied nurses in the current study.

In this regard, UNICEF and WHO (2015), along with a range of partners, have formed a global advocacy initiative to increase political commitment to and investment for breastfeeding as the cornerstone of child nutrition, health and development. The Breastfeeding Advocacy Initiative has three strategic goals; accountability is one of these strategies. Furthermore, UNICEF and WHO (2017) global breastfeeding scorecard was developed by UNICEF in order to tracking progress for breastfeeding policies and program implementation and also intensify the importance of accountability to achieve progress.

Despite variations in approaches to accountability, an accountability regime will always be based on three elements: a clear definition of desirable goals or objectives, the ability to measure and monitor goal achievement and a set of consequences for providers or organizations if achievements regarding goals or objectives are not satisfactory (Denis, 2014). All of these parameters clarify the importance of accountability implication regarding better achievement of ten steps of breastfeeding. For sure, lack of accountability leads to lack of ten steps implementation.

Indeed, lack of accountability may be occurred due to lack of availability of clear protocol or guidelines. Hence, clear protocol communicates each process toward breast feeding initiation and continuation (sustainability). The current study highlights that there is lack of availability of breastfeeding protocol as one of the administrative issues.

The Mothers' discharge form is another administrative theme that reported by the studied nurses in the current study. Mothers who will go outside the maternity hospitals are extremely in needs for support and proper guidance. Hence, such guidance as long term plan must be provided on discharge, take-home healthy message regarding breastfeeding sustainability are documented in El-Shatby hospital in the form of written discharge instruction. Although, the result confirmed that this form is available, the content of the instruction still didn't comply with the goal of breastfeeding sustainability.

These findings confirmed by WHO and UNICEF joint report (2018) which recommended that "mothers should receive practical support to enable them to initiate and maintain breastfeeding and manage common breastfeeding difficulties. As part of protecting, promoting and supporting breastfeeding, discharge from facilities providing maternity and newborn services should be planned for and coordinated, so that parents and their infants receive the appropriate care and have access to supportive resources."

In fact, the ten steps to successful breastfeeding are maternity practices proven to support successful achievement of exclusive breastfeeding (Labbok et al, 2013). The BFHI has been implemented in almost all countries in the world, with varying degrees of success. Inadequate breastfeeding practices significantly impair the health, development and survival of infants, children and mothers (Victora et al, 2016, WHO, 2018). So better understanding of nurses' perception for its challenges is important. Understanding it leads to maintain breastfeeding sustainability and helps obtaining BFHI designation.

\section{SUGGESTED CLINICAL IMPLICATIONS}

The study results and the nurses' responses suggested a lot of recommendations that may help to minimize the barriers that face implementing the ten steps. These clinical implications include;

- Provision of health education about breastfeeding can be given when the nurse provides the needed medication for the mothers and their relatives (visitors as well).

- Access to projected accurate information through Liquid Crystal Display (LCD, screen), video films will help in educating both mothers and their relatives.

- Put a plan to assign the responsibility of initiation of breastfeeding with half an hour through first hour skin to skin contact to specialized nurse at the delivery room.

- Strong managerial support for skin to skin practice at the delivery room is needed. The needed support also to modifying the hospital policy of separating the newborn from their mothers for observation purposes

- Delegate the authority of discharge of well babies from the observation room to the in-patient pediatric physician without waiting the specialists' round. 
- Health education should focus on milk expression (for all cases and not limited on working mothers), correct technique, positioning. Moreover, counseling the mothers during prenatal period especially regarding milk expression and hazards of bottle feeding, on demand feeding and baby hanger cues.

- Have a written infant feeding policy that is routinely communicated to all staff and parents.

- Modifying discharge policy "that permit mothers' discharge alone without their babies" which makes breast milk substitute (BMS) used instead of breastfeeding.

- Inclusion of family members in prenatal breastfeeding health education for new mothers.

- Discharge sheet(form) for the mothers should contain written instruction (in simple language) that encourage and support on-demand feeding (day and night), milk expression, exclusive breastfeeding for the first 6 months, hazards of using bottle and referral to the breastfeeding counseling clinic if needed.

- Instruct the mother to follow written instruction in the discharge form (Giving babies any bottles or pacifiers will be prohibited and the mothers will encourage expressing milk and using cup for feeding). Postpartum mother will be referred to BF consultation clinics.

- It is important for establishing breast feeding continuing education team to train all staff member as well as provision of updated handout offering handout training materials for nursing staff hands on training especially communication and counseling skills as well as encouraging self monitoring frequently.

- It is important to initiate breastfeeding hospital committee that will be responsible for the hospital breastfeeding related issues, breastfeeding coordinators in this committee is expected to reduce inconstancies in practice.

\section{Acknowledgement:}

The authors acknowledge all participant nurses who devote their time to participate in this research, express their gratitude to Mother and Child Friendly care association (MCFC) that support nurses' training for UNICEF/WHO 20 hour course for promoting El-Shataby University Hospital to become a baby friendly Hospital and for valuable facilitation to conduct this research. Many thanks to the hospital manger and head of maternity department, head nurse to obtain their approval for collecting the necessary data from the study setting.

\section{Conflict of interest}

The authors declared that they have no conflict of interest.

\section{Author contribution}

All authors were part of the initial design of the research. They shared in collected and analyzed the data, wrote and edited the final version the text of the manuscript and formatted it and submitted it for publication.

\section{REFERENCES}

Abul-Fadl AM., Faghaly N., ElAzab HS., Rashad M., Mostafa O., AlAttar G., ... and Elkaluby E.(2018) A multicenter survey for monitoring the Baby Friendly Initiative in 6 University hospitals in Egypt (2017-2018): A comparative analysis. Journal of Social Sciences. 06(12):293-306

Alexandria University Hospitals (2013). El-Shatby University Hospital. Retrieved on November 2019. Available at: https://uh-alexu-edu-eg.weebly.com/el-shatby-maternaty-hospital.html

Al Ketbi MI, Al Noman S, Al Ali A, Darwish E, Al Fahim M, Rajah J. (2018). Knowledge, attitudes, and practices of breastfeeding among women visiting primary healthcare clinics on the island of Abu Dhabi, United Arab Emirates. International Breastfeeding Journal volume 13, Article number: 26 (2018).

Al-Madani MM, Abu-Salem LY. (2017). Health professionals' perspectives on breastfeeding support practices. Saudi J Med Med Sci 2017;5:116-23. DOI: 10.4103/1658-631X.204875

Brimdyr K, Cadwell K, Stevens J, Takahashi Y. (2018). An implementation algorithm to improve skin-to-skin practice in the first hour after birth. Matern Child Nutr. 2018 Apr;14(2):e12571. doi: 10.1111/mcn.12571. Epub 2017 Dec 12.

Buontempo M, Busuttil R, Gauci C. (2015). National Breastfeeding Policy And Action Plan - 2015-2020. Health Promotion and Disease Prevention Directorate:Malta.

Cunningham EM, Doyle EI, Bowden RG. (2018). Maternity Nurses' Perceptions of Implementation of the Ten Steps to Successful Breastfeeding. MCN Am J Matern Child Nurs. 2018 Jan/Feb;43(1):38-43. doi: 10.1097/NMC.0000000000000392.

Daniels L , Jackson D. (2011). Knowledge, attitudes and practices of nursing staff regarding the Baby-Friendly Hospital Initiative in non-accredited obstetric units in Cape Town. S Afr J Clin Nutr 2011;24(1):32-38

Denis JL. (2014). Accountability in Healthcare Organizations and Systems. Healthc Policy. 2014 Sep; 10(SP): 89.

Edwards, G, Abdulali, J, Kumar, RR. (2011).Meeting the challenge: implementing the Baby Friendly Hospital Initiative in a culturally diverse country. Pract Midwife.14(6),14-16. 
Fawzi A., Kadry H., Abd Rabo S., Abul-Fadl A.M.A..Challenges Facing the "Updated Baby Friendly Hospital" Program in a Maternity Hospital in Alexandria. MCFC Eg. J. Breastfeeding (EJBF). 2013; 7:53-66.

Genetu, H., Yenit, M.K. \& Tariku, A. (2017). Breastfeeding counseling and support are associated with continuous exclusive breastfeeding from one week to six months of age among HIV exposed infants in north Gondar zone, Ethiopia: a cross-sectional study. Int Breastfeed J 12, 21 (2016) doi:10.1186/s13006-017-0113-1

Jacobs L. (2008). Knowledge, Attitude and Practices of Nursing Staff regarding the baby friendly hospital Initiative in non accredited obstetric units in cape town. Master thesis. Department of School of Public Health, University of the Western Cape.

Kavle JA, Welch PR, Bwanali F, Nyambo K, Guta J, Mapongo N, Straubinger S, Kambale S. (2019). The revitalization and scale-up of the Baby-Friendly Hospital Initiative in Malawi. Matern Child Nutr. 2019;15(S1):e12724. DOI: 10.1111/mcn.12724

Komara, C., Simpson, D., Teasdale, C., Whalen, G., Bell, S., \& Giovanetto, L. (2007). Intervening to promote early initiation of breastfeeding in the LDR. MCN The Journal of Maternal Child Nursing, 32, 117-123.

Labbok M, Taylor FC, Nickel N. (2013). Implementing the ten steps to successful breastfeeding in multiple hospitals serving low-wealth patients in the US: innovative research design and baseline findings. International Breastfeeding Journal volume 8, Article number: 5 (2013)

Lippincott Nursing Education. (2016). The Importance of the Optimal Nurse-to-Patient Ratio. Retrieved on November 2019 Available

at: http://nursingeducation.lww.com/blog.entry.html/2016/11/10/the_importance_ofth-GCAE.html

Liu A, Dai Y, Chen L. (2014). Implementation of International Code of Marketing Breast-Milk Substitutes in China. Breastfeeding Medicine 9(9). DOI: 10.1089/bfm.2014.0053

Mgolozeli SE, Shilubane HN, Khoza LB. (2019). Nurses' attitudes towards the implementation of the MotherBaby Friendly Initiative in selected primary healthcare facilities at Makhuduthamaga Municipality, Limpopo province. Curationis. 2019; 42(1): 1929. doi: 10.4102/curationis.v42i1.1929

Moore K, Peters, L. (2012).Implementing Baby Friendly Practices: Strategies for success.MCN, Vol 37(4), 22833

Nader B, Shahroz F. (2011). Study on the Impact of on the job training Courses on the Staff Performance (A Case Study). Procedia - Social and Behavioral Sciences 29 (2011) 1942 - 1949

Pérez Escamilla R, Martinez J, Segura Pérez S. (2016). Impact of the Baby friendly Hospital Initiative on breastfeeding and child health outcomes: a systematic review. Maternal and child Nutrition Journal, Volume12, Issue3, July 2016: 402-417.

Ratnasari D, Paramashanti BA, Hadi H, Yugistyowati A, Astiti D, Nurhayati E. (2017). Family support and exclusive breastfeeding among Yogyakarta mothers in employment. Asia Pac J Clin Nutr 2017;26(Suppl 1): $: 31-S 35$

Schmied, V., Gribble, K., Sheehan, A., Taylor, C., \& Dykes, F. C. (2011). Ten steps or climbing a mountain: a study of Australian health professionals' perceptions of implementing the baby friendly health initiative to protect, promote and support breastfeeding. BMC health services research, 11, 208. doi:10.1186/1472-696311-208

Schoonenboom, J., \& Johnson, R. B. (2017). How to Construct a Mixed Methods Research Design. Kolner Zeitschrift fur Soziologie und Sozialpsychologie,69(Suppl 2), 107-131. doi:10.1007/s11577-017-0454

Smith J, Cattaneo A, Iellamo A, Javanparast S, Atchan M, Gribble K, Hartmann B, Salmon L, ... and ElliottRudder M. (2018). Review of effective strategies to promote breastfeeding: An evidence check rapid review brokered by the Sax Institute for the Department of Health. Australia: Sax Institute for the Department of Health.

Smith P, Moore K, Peters, L. (2012). Implementing Baby Friendly Practices: Strategies for success. MCN, Vol $37(4), 228-33$

Stolzer JM and Hossain SA.(2014). Breastfeeding Education: A Physician and Patient Assessment. Child Development Research. Volume 2014, Article ID 413053, 6 pages. http://dx.doi.org/10.1155/2014/413053

Taylor C, Gribble K, Sheehan A, Schmied V, and Dykes F. (2011). Staff Perceptions and Experiences of Implementing the Baby Friendly Initiative in Neonatal Intensive Care Units in Australia. JOGNN, 40, 25-34; 2011. DOI: 10.1111/j.1552-6909.2010.01204.x

The American College of Obstetricians and Gynecologists. (2016). Optimizing Support for Breastfeeding as Part of Obstetric Practice. Retrieved on November 2019. Available at: https://www.acog.org/Clinical-Guidanceand-Publications/Committee-Opinions/Committee-on-Obstetric-Practice/Optimizing-Support-for-

Breastfeeding-as-Part-of-Obstetric-Practice?IsMobileSet=false

Turner-Maffei C, Cadwell K, editors. Overcoming Barriers to Implementing the Ten Steps to Successful Breastfeeding. Sandwich, MA: Baby-Friendly USA, 2004.

UNICEF / WHO. (2015). Breastfeeding Advocacy Initiative For the best start in life. UNICEF Indonesia Communication Unit: Indonesia. 
UNICEF. (2015). Health. Retrieved on November 2019. Available at: https://www.unicef.org/egypt/health.

UNICEF/WHO. (2016). WHO/UNICEF Baby-Friendly Hospital Initiative (BFHI) Congress. Retrieved on November 2019. Available at: https://www.who.int/life-course/news/events/baby-friendly-hospitals2016/en/

UNICEF / WHO. (2017). Global Breastfeeding Scorecard, 2017: Tracking Progress for Breastfeeding Policies and Programmes. WHO: Genève, 2017.

UNICEF, UK. (2019). The baby friendly initiative: Skin To Skin. Retrieved on December 2019. Available at: https://www.unicef.org.uk/babyfriendly/baby-friendly-resources/implementing-standards-resources/skin-toskin-contact/

United Nations. Sustainable Development Goals: 17 goals to transform our world (http://www.un.org/ sustainable development/sustainable-development-goals/, accessed 7 March 2018).

United Nations Children's Fund. UNICEF data: monitoring the situation of children and women. Access the data: infant and young child feeding (http://data.unicef.org/topic/nutrition/infant-and-young-childfeeding/, accessed 7 March 2018).

Victora CG, Bahl R, Barros AJ, França GV, Horton S, Krasevec J et al. Breastfeeding in the 21st century: epidemiology, mechanisms, and lifelong effect. Lancet. 2016;387(10017):475-90. doi:10.1016/S01406736(15)01024-7.

Weddig (2011). Improving breastfeeding initiation practices of registered nurses through online theory-based education. dissertation. Colorado State University

Wieczorek CC, Schmied H, Dorner TE, Dür W. (2015). The bumpy road to implementing the Baby-Friendly Hospital Initiative in Austria: a qualitative study. Int Breastfeed J. 2015; 10(1):3.

World Health Organization.(2003) Global Strategy for Infant and Young Child Feeding Geneva Switzerland Retrieved 2019 at: https://www.who.int/nutrition/topics/global_strategy_iycf/en/

WHO.(2018a). Marketing of breast-milk substitutes: national implementation of the international code, status report 2018. Department of Nutrition for Health and Development: Switzerland.

WHO.(2018b) Ten steps to successful breastfeeding (revised 2018). Retrieved on December 2019. Available at: https://www.who.int/nutrition/bfhi/ten-steps/en/

WHO.UNICEF (2018). Implementation guidance: protecting, promoting and supporting breastfeeding in facilities providing maternity and newborn services - the revised Baby-friendly Hospital Initiative. WHO Library: Geneva

Yang S, Burns E, Salamonson Y, Schmied V. (2019). Expectations and experiences of nursing students in supporting new mothers to breastfeed: A descriptive qualitative study. J Clin Nurs. 2019;28:2340-2350. DOI: $10.1111 /$ jocn.14836. 